\title{
GCU
}

Glasgow Caledonian

University

University for the Common Good

\section{Marketing authorization procedures for advanced cancer drugs: exploring the views of patients, oncologists, healthcare decision makers and citizens in France}

Protiere, Christel; Baker, Rachel; Genre, Dominique ; Goncalves, Anthony ; Viens, Patrice

Published in:

Medical Decision Making

DOI:

$10.1177 / 0272989 \times 17691953$

Publication date:

2017

Document Version

Author accepted manuscript

Link to publication in ResearchOnline

Citation for published version (Harvard):

Protiere, C, Baker, R, Genre, D, Goncalves, A \& Viens, P 2017, 'Marketing authorization procedures for advanced cancer drugs: exploring the views of patients, oncologists, healthcare decision makers and citizens in France', Medical Decision Making, vol. 37, no. 5, pp. 555-566. https://doi.org/10.1177/0272989X17691953

\section{General rights}

Copyright and moral rights for the publications made accessible in the public portal are retained by the authors and/or other copyright owners and it is a condition of accessing publications that users recognise and abide by the legal requirements associated with these rights.

Take down policy

If you believe that this document breaches copyright please view our takedown policy at https://edshare.gcu.ac.uk/id/eprint/5179 for details of how to contact us. 
Marketing authorization procedures for advanced cancer drugs: exploring the views of patients, oncologists, healthcare decision makers and citizens in France $^{1}$

Christel PROTIERE, PhD ${ }^{1}$, Rachel BAKER, $\mathrm{PhD}^{2}$, Dominique GenRe, PhD ${ }^{3}$, Anthony GONCALVES PhD ${ }^{4}$, Patrice VIENS, $\operatorname{Pr}^{4}$

1. Aix Marseille Univ, INSERM, IRD, SESSTIM, Sciences Economiques \& Sociales de la Santé \& Traitement de l'Information Médicale, Marseille, France.

2. Yunus Centre for Social Business and Health - Glasgow Caledonian University

3. Institut Paoli Calmettes, Direction de la Recherche Clinique et de l'Innovation, F-13009, Marseille, France

4. Institut Paoli Calmettes, Department of Molecular Oncology, and CRCM, Inserm, U1068; Aix-Marseille Université, UM 105; CNRS, UMR7258, F-13009, Marseille, France

\begin{abstract}
The past decades have seen advances in cancer treatments in terms of toxicity and side effects but progress in the treatment of advanced cancer has been modest. New drugs have emerged improving progression free survival but with little impact on overall survival, raising questions about the criteria on which to base decisions to grant marketing authorizations and about the authorization procedure itself. For decisions to be fair, transparent and

\footnotetext{
${ }^{1}$ Financial support for this study was provided entirely by a grant from La Ligue Nationale contre le Cancer (The national league against cancer, contract number RAB09001AAA). The funding agreement ensured the authors' independence in designing the study, interpreting the data, writing, and publishing the report.
} 
accountable, it is necessary to take account of the views of those with relevant expertise and experience.

We conducted a Q-study to explore the views of a range of stakeholders in France, involving: 54 patients (18 months after diagnosis); 50 members of the general population; 27 oncologists; 19 healthcare decision makers; and 2 individuals from the pharmaceutical industry.

Three viewpoints emerged, focussing on different dimensions entitled: I 'Quality of life (QoL), opportunity cost and participative democracy', II 'QoL and patient-centeredness' and III 'length of life'. Respondents from all groups were associated with each viewpoint with the exception of healthcare decision makers, who were only associated with the first one.

Our results highlight the plurality in the views of stakeholders and emphasize the need for transparency in decision processes and the importance of re-evaluation of treatments for all three viewpoints. In the context of advanced cancer, our results suggest that QoL should be more prominent amongst authorization criteria, as it is a concern for two of the three viewpoints.

$\mathrm{N}=237$

Key Words: Marketing authorization; Q-methodology, advanced cancer; efficacy criteria; societal perspectives; health policy 


\section{Introduction}

The past decades have seen advances in cancer treatments that have reduced toxicity and side effects. Despite these successes, there have been only modest advances in the treatment of advanced cancer [1,2]. Treatments are generally not curative, and survival benefit is usually measured in weeks or months $[3,4]$. Recently, new drugs have been developed which improve progression free survival but which have little impact on overall survival (such as Bevacizumab [5]). The emergence of such (typically high cost) drugs raises questions about the criteria that should be used in marketing authorization decisions and related ethical, economic and political issues. Should drugs for advanced cancer, which promise only modest extensions to life, be authorised for coverage from the public purse?

Before a medicine can be sold it must have a license called a marketing authorization (MA).

At the European level, once a drug has been recommended by the Committee for Medicinal Products for Human Use, MA is granted by the European Medical Agency (EMA). The decision to authorize medicines is based on three criteria relating to quality, safety and efficacy. The assessment of quality covers all aspects of the industrial production process. Safety is assessed on the basis of all research conducted during pre-clinical development. The efficacy criterion takes account of all of the clinical trials conducted on humans. Moreover, MA is granted if the new drug presents a benefit/risk ratio (established by the EMA) at least equivalent to the products already offered for sale - an additional 'non-inferiority' criterion (see [6] for a description of the procedure). These four criteria have been debated by physicians, with particular focus on efficacy (in relation to the choice of the relevant 
endpoint) and non-inferiority [2,7-11]. In a second step, which is specific to each European Union member state, the coverage and the cost of the treatment are established.

Davis (2015) has established a comprehensive review of the literature about the growing importance of the pharmaceuticalization in the context of advanced cancer, showing the increased use of aggressive, and sometimes inappropriate, drugs and questioning the relevancy of the MA process [4]. However, to our knowledge, no survey has directly exploring, the views of stakeholders in relation to the procedures used to determine MA. Their views are crucial if such decisions are to be fair, transparent and accountable, and if they are to reflect the views of those with relevant expertise and experience. This research contributes to the debate surrounding MA decisions by exploring the perspectives of all stakeholders in France: health care decision makers (concerned with resource allocation and the promotion of best practice); medical oncologists and cancer patients (the main actors in delivery of care and the beneficiaries of care); and the general population (potential patients and citizens who contribute to the funding of health services and medicines). Members of the pharmaceutical industry were also included in our sampling frame, as they are involved in the research and production of treatments, but recruitment was unsuccessful, as will be reported. Because all of these stakeholders occupy different positions in the process, they are likely to have different points of view and different priorities.

This paper reports the findings of a study using $Q$ methodology to elicit stakeholders' viewpoints about the dimensions at stake in determining $M A$ and about the processes used to grant MA (including whether or not the cost of the treatment should be considered in the MA procedure). 


\section{Methods}

\section{Q methodology}

Q-methodology is designed to explore the nature of subjective viewpoints [12]. Developed by William Stephenson, Q methodology comprises qualitative and quantitative methods [1316] and has been applied in health related studies on topics such as understanding pain [17], the concept of quality of life [18], patients' preferences toward the treatment of hypertension [19], informal caregivers' attitudes [20] or eliciting the public's views on health care resource allocation [16]. Q methodology was chosen because it allows the researcher to compare the accounts of respondents based on their response to a common set of propositions; it enables a holistic analysis [13,21]; it allows the emergence of counter intuitive results and it assists respondents in expressing their views by ordering a set of carefully selected statements.

In Q studies respondents rank order a set of statements of opinion about a topic, on a grid, labelled according to a subjective dimension such as 'agreement/disagreement'. This rank ordering, called a Q-sort, is obtained for each respondent. Factor analysis performed on the Q-sorts results in factors that represent shared viewpoints. In other words, in Qmethodology, each factor obtained from the factor analysis represents a viewpoint shared by a group of respondents who have ordered the set of statements in a similar fashion. If each individual has a different viewpoint on the question, their Q-sorts will not correlate; if significant clusters of correlation exist, they can be factorized, described as common viewpoints, and individuals are mapped to a particular factor [20]. Each viewpoint is described by a distinct ordering of statements, which is interpreted holistically so that 
statements are not interpreted in isolation, but collectively and relative to the position of the other statements.

\section{The statements (Q-set)}

Following a review of the literature, 21 semi-structured interviews were conducted with five members of the patients' committee from the Institut Paoli-Calmettes (IPC) the regional cancer centre in Marseille; five medical oncologists from IPC and Institut Sainte Catherine in Avignon; three healthcare decision makers from HAS and the French National Cancer Institute; four members of the pharmaceutical industry; and four members of the general population. A first set of 54 candidate statements was extracted from the interview transcripts, [22] selected because they represented distinct statements of opinion in relation to the topic of study. To help us reducing the set of statements, this first set was submitted to the medical oncologists and patients who had been interviewed, and to five (new) members of the general population. A final Q-set of 34 statements was selected (and reworded in accordance with the comments) in order to cover the range of issues at stake, that could be classified onto four dimensions (See table 2 for a list of the statements): quality of life (QoL) and side effects; clinical gain (duration of the life extension); cost of treatment and MA procedure (see table $A 1$ in appendix for the links between statements and the four dimensions). The final Q-set was pilot tested with the three co-authors from IPC (DG, AG, PV), 5 new members of the general population and four (new) patients from IPC. We did not identify any concerns about comprehension of statements from our pilot study.

\section{Respondents (P-set)}

Selection of respondents in $Q$ studies has much in common with qualitative, purposive sampling techniques and respondents are selected who will have strong views, relevant 
experiences and different viewpoints from one another. In this study participants were recruited from 5 groups: non metastatic cancer patients, members of the general population, medical oncologists, health decision makers, and representatives from the pharmaceutical industry. As a guide to the size of the $\mathrm{P}$ set, Brown suggests that $40-60$ respondents is more than likely adequate [23]. We have chosen a relatively large sample (in $Q$ terms) to retain the option to analyze the data for each respondent subgroup as well as pooling the data for the whole sample. On examination all the viewpoints elicited by group are highly correlated with one of the viewpoint from the pooled data (see figure A1 in appendix), hence we present results based from the whole sample.

The resulting sample includes more patients and members of the general population than oncologist and health care decision makers. We anticipated that professionals would have more 'established' viewpoints, ones which they may have previously articulated, and so (partly to reduce the time and cost of data collection) we aimed to collect 15 to $20 \mathrm{Q}$-sorts from medical oncologists and from health decision makers, 10 to 15 from members of the pharmaceutical industry and 50 from patients and members of the general population ${ }^{2}$ :

- 54 patients (PA), in remission at 18 months of their diagnosis were recruited at IPC. Nonmetastatic cancer patients were identified, by the clinical research and innovation department, from consultation lists. They were informed of the subject and the conditions of the study by mail. If they did not wish to participate, they could indicate non-consent by sending a reply in a pre-paid envelope, otherwise they were met by a psychologist on the day of their consultation.

\footnotetext{
${ }^{2}$ It is worth considering whether this would have an effect on the factor solution. In Q methodology, once factors are identified, subsequent similar Q-sorts serve only to substantiate the account represented by that factor. For that reason, we don't expect the factor solution is altered significantly by this sampling strategy, unless we have inadvertently, systematically excluded a group of respondents with a different viewpoint...
} 
- 50 members of the general population (POP) were recruited in a municipal library (named Alcazar, and located in the center of Marseille, France, which allowed us to recruit people belonging to a large sample of different socio-professionals categories). Participants were informed of the research using flyers and a poster at the entrance of the library asking for volunteers to participate.

- 27 oncologists (ON) were recruited via the oncologists' directory and the Centres Against Cancer websites.

- 19 health decision makers (HDM) from public organisations with some responsibility for allocation of resources and promotion of good practice (regional health agencies and the HAS).

- Only 2 representatives of the pharmaceutical industry (IND) agreed to participate despite asking the LEEM (the French Pharmaceutical Companies Association) to recruit respondents on our behalf.

The professionals (ON, HDM and IND) were sent an email informing them of the research and providing them with a link to the online questionnaire.

\section{Q-sort administration}

The Q-sort was administered face to face using a computer interface for PA and POP, and online for the professionals. No remuneration was offered to any of the participants to the survey.

Q-sorts require participants to order and prioritize each statement in relation to every other statement. First, respondents were asked to sort the statements, presented to them in random order, into three piles: agree, disagree and neutral. Respondents were then guided through a process of ranking the statements, onto a response grid, from the ones they agree 
with the most to the ones they disagree with the most. The grid was designed in a quasinormal shape, with a fixed number of items placed under each scale point (see figure 1). The two statements they agreed with the most were placed under $(+4)$ and the two they disagreed with the most were placed under $(-4)$, and so on, working towards the centre of the grid, until all statements were placed. Once the sort was complete, respondents were invited to examine and adjust it if they wished. They were then asked to provide open-ended comments to explain their rationale for the choice of the four statements placed at the extremes.

We used FlashQ (http://www.hackert.biz/flashq/home/) a dedicated online Q-sorting tool.

\section{Data-analysis}

The Q-sorts were analyzed using a dedicated software package PQMethod 2.35 which facilitates $Q$ factor analysis and interpretation (see the $P Q$ manual for details http://schmolck.userweb.mwn.de/qmethod/downpqwin.htm). The first step in $Q$ factor analysis is the calculation of a correlation matrix, which presents the degree of similarity between individuals' $\mathrm{Q}$-sorts. The objective of $\mathrm{Q}$ factor analysis is to identify a limited number of similar ways in which respondents have completed their Q-sorts (i.e. rank ordered the statements). Q-sorts with higher loadings are given more weight in the idealized Q-sort since they better exemplify the factor. In this way each factor (representing a shared viewpoint) is represented by a single, idealised Q-sort [18]. This idealized Q-sort represents the Q-sort of a person who would be correlated 100 percent with that factor. Confounded Qsorts (i.e. those with significant loading on more than one factor) were not used in the calculation of the idealized $\mathrm{Q}$-sort (see table $\mathrm{A} 1$ in appendix for more details) $[12,23]$. Interpretation of each viewpoint is based on the idealized Q-sorts together with respondents' 
comments on their Q-sorts. The salient statements, at the extreme poles of the grid, are important to interpretation as are significantly 'distinguishing statements' that are placed differently between factors. The interpretation is not made statement by statement, but rather aims to present a holistic interpretation of each viewpoint.

There are several approaches to factor extraction and rotation used by $\mathrm{Q}$ methodologists, and debate about which is more appropriate $[12,24]$. The objective of the rotation is mainly to facilitate interpretation of the factors [25]. Manual rotation allows the analyst to examine the data from a graphical perspective, not to alter the correlations between Q-sorts, but to examine the relationships between Q-sorts from different angles. Varimax rotation is also used by $Q$ methodologists; often because this is the statistical procedure most often used in standard factor analysis and maximizes the variance explained by the rotated factor solution [12]. The two rotation methods have advantages and disadvantages. In this analysis several combinations of extraction (centroid or principal component analysis) and rotation (manual or varimax) were examined and compared.

Manual rotation is generally guided by 'hunches' that emerge about the data, the subject matter or about participants' accounts [26] or may be guided by theoretical reasoning [27]. Where several solutions are available to the analyst, selection of a 'best' solution is often an iterative process, following up on hunches and consulting both qualitative and quantitative information [26]. In this case we were interested in examining the patterning of different respondent types across factors and, in particular, to manually rotate factors in relation to respondents in the smallest group (from the pharmaceutical industry) to examine the factors from that perspective. Because this group was small we expected that the account might be lost in a purely statistical solution, which was the case. Although one IND, loaded on the second factor in the unrotated matrix, it was no longer the case with the centroid varimax 
solution. Following graphical examination of the factors in relation to all of our respondent groups we conducted a fairly straightforward rotation in an attempt to maximize the number of uniquely correlated ("pure") Q-sorts, preserving the loading of the IND Q-sort. The best solution was obtained by rotating factors F1 and F2 -25 degrees, and rotated factors F1 and F3 +5 degrees. To test the robustness of our results, we compared the results of our manually rotated solution with the solution generated by centroid followed by a varimax rotation, which revealed statistical comparable results ${ }^{3}$.

We then report the solution of principal component analysis followed by manual rotation which was preferred on grounds of interpretability and consistency with respondents' accounts (including viewpoints elicited by group - see Fig. A1) and secondly because it allowed the most respondents $Q$ sorts to be purely associated with a single factor (in other words it was the most interpretable solution with the most pure loadings) without sacrificing the variance explained compared with the varimax alternative.

\section{Results}

The final participant sample was composed of 146 participants. Q-sorts from two PA and four POP were excluded, because they stated that the rank given to one of the statements at the extreme (+/-4) was positioned in error. The sample consisted of 72 women and 74 men with mean age 49 years (see Table 1 for a description of the sample).

Our data supported a maximum of a four-factor solution, on the basis that each factor should include at least two non-confounded loaders (individuals with significant correlation with

\footnotetext{
${ }^{3}$ The correlation matrix shows that: PCA F1 is correlated with CEN F1 (0.94), PCA F2 is correlated with CEN F3 (0.71) and PCA F3 is correlated with CEN F2 (0.84). Moreover, the manual rotation accounted for more defining variables (97 vs 87 ), with similar percentage of explained variance $(44 \%$ vs $43 \%)$ and resulted in lower correlation coefficient between factors.
} 
only one factor and not the others). As the four factor solution added only $3 \%$ to the total variance explained (47\% vs. $44 \%$ ) and presented less distinction in the content of each factors (higher correlation coefficients between factors, see. figure 2) the three-factor solution was selected. With this three viewpoint solution 97 respondents were uniquely associated with a single viewpoint and 16 respondents were confounded), the 33 remaining respondents were not correlated with any of the three factors (Table A2). In what follows, we present a description of the respondents and then we describe each viewpoint they are associated with. Rankings of the statements are provided in abbreviated form, $(\mathrm{s} 31+4)$ means that statement 31 is ranked in the +4 position (agreed the most) and (s29 -4) means that statement 29 is ranked in the -4 position (disagreed the most) for the viewpoint in question. The statements and respondents' comments have been translated from French and quotes are provided to illustrate or clarify interpretations (respondent ID number/ type of participant is indicated).

\section{Viewpoint 1: Quality of life, opportunity cost and participative democracy}

Seventy-two respondents' Q-sorts were significantly correlated with viewpoint 1: 16 HDM, 18 ON, 13 PA, 25 POP.

This first viewpoint regards QoL as more important than length of life (s27 -4, s29 -4, s18 -3, s33 +3, s22 +1), and an effective treatment is one which allows the patient to live a life comparable to their life before the disease $(s 2+2, s 23+1)$. So, for the HDM83 "Quality of life must be included as a major criterion, especially for a drug that improves survival very little". The emphasis on QoL is coupled with a concern about MA procedures. For viewpoint 1, as for the two others, it is important that outcomes of the MA committee are transparent and made public $(s 31+4)$ and that treatments are regularly re-evaluated after their launch on the 
market in order to judge their efficacy and their tolerance in general practice $(s 28+4)$. PA36 states that "Transparency is essential in democracy". There is some support in this account for granting temporary authorization, and delivering definitive MA only after the treatment has been proven over time and among different populations $(s 11+2)$. The procedures of the MA committee should also take into account the views of patients through a patient representative $(s 6+3)$, patients' reports of the impact of side effects on their everyday life $(s 34+3)$, and to a lesser extent the views of the general public $(s 7+2)$. This viewpoint is hence concerned with a form of participative democracy which takes account of the views of those people directly affected by decisions as well as the points of view of citizens.

Lastly, the position taken on the cost of treatments distinguishes this viewpoint from the two others. Participants associated with this viewpoint believe that MA should also take into account the impact of coverage decisions for society more broadly and the benefits of treatments should be weighed up against alternative uses of resources (s19 -3, s4 -2, s17 -2). In other words the opportunity cost for society, and not only the needs of the patients, should be considered as part of the MA procedure $(s 26+2)$. As such the MA committee takes the role of agents of the citizen. This is an important distinction, which we return to in the discussion.

\section{Viewpoint 2: Quality of life and patient-centredness}

Eighteen respondents were significantly associated with this viewpoint: 3 ON, 11 PA, 3 POP, 1 IND.

The core of this viewpoint is patient-centred. Like viewpoint I, QoL is of greater concern than length of life (s18 -4, s27 -3, s29 -3, s32 +2, s33+2) as explained by PA82, "On the contrary, I think that in case of an incurable cancer, the point is the quality of the remaining life". In 
contrast with viewpoint 1, importance is placed on life expectancy gains. Cost, on the other hand, is not a central issue for viewpoint 2. Consider, for example, the position of statement 14 at +4 (-1 and 0 for viewpoints 1 and 3 respectively), summed up by the comments of one patient, "Life has no price. Society must contribute to cost of care" (PA108). The cost of treatments to society is not an issue which should be considered during the MA procedure (s26 -4) and this view is evident in the placing of all statements describing a trade-off between a clinical gain and cost of treatments (s14 +4, s4 +2, s17 +2). ON60 "the cost of drugs should not affect the improvement of quality of care [...] however, we have to be reasonable".

As in the previous viewpoint, MA procedures should include treatment re-evaluation $(s 28+4)$ and the need for transparency $(s 31+3)$ is emphasized. Patients' reports of the impact of side effects on their everyday life is seen as important $(s 34+3)$, but views about the inclusion of a patient representative as part of the MA committee are more equivocal $(s 6+1)$ and considering the view of the general population is not relevant (s7 -2). Lastly, temporary MA is of some appeal for this viewpoint as a means to accelerate access to promising treatments $(\mathrm{s} 24+3)$ but not as a means to impose restrictions on a definitive MA (s11 0).

\section{Viewpoint 3: Length of life}

Eight respondents loaded significantly on this viewpoint: 4 ON, 2 PA, 1 POP.

This viewpoint is characterized by the priority given to any treatments that result in life extension and a refusal to define a minimum threshold in terms of survival gain required for MA (s8 -4, s10 -4, s9 -3, s15 -2, s16 -2). Even a gain of 1 month is considered sufficient $(s 5+2)$ regardless of the cost to society (s19 0). POP28 commented that, "survival gain should not be rigid criteria to grant MA" and ON61 claimed, "Patients prefer benefiting from a treatment 
even if the gain in expected survival is small. In this case 6 months is an average gain, some patients will benefit from longer survival, for instance 2 years!". There is also importance placed on increasing the range of available treatments, especially when few treatments are available $(s 25+4)$ and to the rapidity in access to the new treatments, using temporary MA $(s 24+3)$.

In contrast with the two other viewpoints, QoL does not prevail over length of life (s33 -3, s23 -2), and a gain in survival is preferred even if it implies a decrement in QoL (s12 +2 , s18 +1 , s29 +1). A treatment that decreases the risk of disease progression is appropriate even if it has negative impact on family, social and professional life $(s 30+3)$, on thinking skills, concentration and memory $-\mathrm{s} 3+1)$. The idea that an effective treatment is a treatment which allows the patient to live a comparable life as the one before the disease is seen as unrealistic (s2 -3).

Although views concerning transparency $(s 31+4)$ and re-evaluation $(s 28+3)$ are shared with viewpoints 1 and 2, taking into account the patient's point of view during the MA process is not so important for this third viewpoint (s34 0, s6 -1), and including a representative of the public in MA process is not relevant (s7 -2). Lastly, the question of treatment cost is not a concern in this viewpoint and none of the statements concerned with cost were placed at the extremes of the grid (+/- 4 and 3$)$.

\section{Discussion}

The criteria used to make decisions to grant MA for medicines raises issues of ethical, political and economic debate, especially in the context of drugs for advanced cancer. We contribute to this debate with research based on the opinions of 146 stakeholders: non metastatic cancer patients at 18 months after their diagnosis, oncologists, health users 
(citizens) and health decision makers. All of these stakeholders have different roles, knowledge, experiences and interests, and thus potentially have different points of view. To our knowledge this is the first survey eliciting the views of a large range of stakeholders, including a substantial cancer patient group [28]. We have shown not only that different views exist, but also how they emerge from these different stakeholder groups, revealing that respondents' membership or allegiance with groups as defined here is not a good predictor of point of view, but that the views of HDMs are clustered around one viewpoint and not the others. Although we hoped to include the views of those employed in the pharmaceutical industry, only two people agreed to participate (of the 10 to 15 expected). It's difficult to explain the reason for their refusal to participate, despite our attempts to recruit respondents through the LEEM. One explanation could be a sensitivity amongst potential respondents with respect to the image of the pharmaceutical industry because of some recent cases $[29,30]$.

Q Methodology provides a framework for enabling respondents to rank a large number of statements, and thereby express subjective opinions about complex issues. It not only helps reveal how respondents feel about the issues raised by a topic but also identifies the relative significance that they attach to those issues. An advantage of this approach over more conventional qualitative methods is the capacity to present a set of statements to all respondents to consider. Using other methods it is possible that some dimensions may be missed because each sub-population is confronted only with their own perceptions of the topic $[16,22]$. This is illustrated by contrasting our $Q$ study findings with the findings of a previous qualitative phase of research, where we showed variability of the significations associated with the criteria of efficiency of cancer treatments according to the sub populations [16]. 
Whilst $Q$ studies are effective methods for eliciting and describing shared viewpoints on a subject, it is not a means of generating information about the proportion of population who agree with these views $[15,22]$. Three viewpoints emerged from our analysis: the first concerned with quality of life, opportunity cost and participative democracy; the second focussed on quality of life and patient-centredness and the third centrally concerned with length of life. Given the populations surveyed it is perhaps unsurprising that elements of these three viewpoint emerged. However, the distribution of 'respondent-types' across the three viewpoints is interesting. The QoL, opportunity cost and participative democracy viewpoint is held by respondents in all four sub-populations. Viewpoints two and three are shared by oncologists, patients and the public, no health decision makers loaded significantly on these viewpoints. Thus, whilst the views of experts/professionals were not misaligned with the views of patients and the general population, health care decision makers are closely aligned only with one viewpoint. Oncologists and patients, who might be expected to have strong opinions, were associated with all three viewpoints. Our failure to recruit members of the pharmaceutical industry means that we are unable to draw any conclusions about how their views align with the views of others, but we have retained two industry respondents' data in our analysis, one of whom was associated with the second viewpoint.

There may be some limitations in our methods that relate to the selection of the statements. In $Q$ studies it is expected that respondents who are associated with different viewpoints might interpret different statements in different ways. Wording is often more loosely constructed than in survey research, since it is the collection of all statements in a specific order that reveal the different ways of seeing an issue, and which we attend to when interpreting viewpoints. Despite efforts to cover the range of issues, and to find balance such that respondents with different views on the topic can find a means to express those views 
through the $Q$ set, some statements might be difficult to understand, might overlap too much or could limit respondents expression of their views in some ways that we are not aware of. Our pilot work indicated that statements were well understood.

Turning to the narratives, and what they might mean for policy and practice, issues of consensus were identified across the viewpoints. There was agreement about the importance of re-evaluating treatments after their launch on the market, to judge their efficiency and their tolerance in general practice $(s 28:+4+4+3)$. The importance placed on transparency (concerning the outputs of the MA committee and the wish to make them public) was also a major concern across all viewpoint $(s 31:+4+3+4)$. Our findings suggest that simply making the MA committee reports publicly available (as they have been since 2006) is not sufficient. ON79 expressed a "wish to better understand the reasons of an acceptance or a refusal of a MA". More widely, this strong interest in transparency might be related to the need to restore confidence as illustrated by the so-called French Sunshine Act (Decree $n^{\circ}$ 2013-414 devoted to bring transparency [32]) and to wider pressure for a more ethical economy.

The beginnings of a response, balancing the need for rapid access to drugs for new indications and the lack of information on their benefit-risk ratio, has been proposed through the "temporary recommendations for use" (TRUs; decree number 2012-743) [33], which, as shown by our results, is consistent with the expectations of ON, PA and POP from viewpoints $2 \& 3$. This decree aims to regulate off-label drug use, but could potentially be extended to all new drugs, as hoped for by all stakeholders associated with viewpoint 1 . 
Applications for MA are evaluated according to quality, safety and efficacy criteria, that is to say, according to "hard", scientific criteria. Our survey has shown, in the context of advanced cancer, the importance attached to QoL. Because it is essentially subjective and multidimensional [18] QoL is more difficult to evaluate. QoL is considered essential by respondents associated with viewpoint 1 "QoL, opportunity cost and participative democracy" and viewpoint 2 "QoL and patient-centred" but not by viewpoint 3 "the length of life-centred". However, it is interesting to note that some professionals stated, during the qualitative step, that extending length of life automatically implied an improvement in QoL. This result raises questions about the appropriate efficacy criteria to be considered in authorising a new drug for advanced cancer. Steer et al. (2001) argue that in patients with advanced disease therapy is not usually curative and, as such, endpoints associated with palliative care are more relevant. This turns the focus towards quality of life (rather than objective measures of, for example, tumour response rate and its duration) as a more relevant endpoint. This result is also in line with research by Silvestri et al. (1998) who have shown that a higher proportion of non-small lung cancer patients choose chemotherapy for a reduction of symptoms, without prolonging life, rather than for a survival benefit [34]. It is important to consider means to better take account of QoL as an endpoint during the MA process for medicines for advanced cancer. One step could be to focus on time to tumor progression free survival if a link between QoL and progression free survival is confirmed [8]. Another approach could be to define more specific criteria for instance according to the length of expected median survival time [2].

Beyond these criteria for authorization, the new product must have a benefit risk ratio at least equivalent to the products already on the market. It is notable in our research that one of the consensus statements $(s 1:-1-1-1)$ indicates that respondents were rather against (or 
neutral) about this " non inferiority » criteria, which is illustrated by the comment of HDM88. "There is no interest to increase the number of options available, if there is no therapeutic gain". The requirement to demonstrate added value for all new drugs, or at least to demonstrate that benefits outweigh side effects has already been discussed in the literature $[29,35]$.

One issue that distinguishes the viewpoints from each other is whether the cost of treatments should be considered during the MA procedure (s26: $+2-4-1)$. Although from a pragmatic perspective incorporating 'price' as part of the MA procedure would be difficult at the European level (at least for the moment because of differences between countries in approaches to the funding and provision of health care) the question remains important. The issue of resource allocation and priority setting is addressed by HDM40 "Need to include public health ethics and not individual ethics. When a drug is on the market, the physician has to propose it to the patient, to inform him of the benefits and side effects and it is not his job to take cost for society into account. The cost that society is prepared to incur for a year adjusted on quality of life must be set upstream." This point is central for the viewpoint 1 "QoL, opportunity cost and participative democracy" with the rejection of every statement supporting the provision of treatments which generate health gains whatever the cost for the society as declared by POP60 "It's crucial to calculate the relationship between the benefit of the drug and its cost for society, i.e. the cost of survival". The fact that the two other viewpoints do not considered the question of cost may not necessarily mean they reject the reality of a budget constraint, but perhaps that they do not prioritise this dimension as much as the others. In a $Q$ study in the UK, investigating the relative value of life extension for people with terminal illnesses, a factor that rejects cost as a criteria has also be identified 
[28]. In their discussion of their findings McHugh et al. (2015) argue that respondents did not challenge the reality of a fixed budget, but thought that life-extending treatments for patients with terminal illnesses would be affordable if government distributed resources more appropriately "For example, some interview respondents suggested reallocating resources from other areas of government spending (e.g. defence), cutting waste in the NHS, or greater regulation of pharmaceutical industry pricing"p13.

\section{Conclusion}

Our results emphasize the need for transparency and re-evaluation of treatments after they have received MA. These two issues were important to respondents associated with all the three points of view, and views expressed by all categories of respondent.

Our findings also suggest that, in the context of advanced cancer, QoL is important, although not supported by viewpoint 3 , which is primarily concerned with survival gain, showing the complexity of the topic and the need to consider the diversity found in subjective opinion on this topic as well as issues of consensus.

\section{Acknowledgement}

The authors wish to thank all the participants for their time and their comments; Alcazar, a public library in Marseilles for allowing us to meet and to interview members of the general population, be3 (http://be3.fr) for the conception of the online and offline webquestionnaire.

The authors also wish to thank Stéphanie Blois Da Conceição and Noémie Jeannin for their management of the project and the interviews they have conducted, as well as the Department of Clinical Research and Innovation from IPC for their logistic help. The authors also wish to thank Gilles Bouvenot for his helpful comments the anonymous reviewers for helpful comments which have helped us to improve this paper.

$\mathrm{N}=5819$ 


\section{References}

[1] Matheson A. Five steps for structural reform in clinical cancer research. Am J Public Health 2010;100:596-603.

[2] Sobrero A, Bruzzi P. Incremental advance or seismic shift? The need to raise the bar of efficacy for drug approval. J Clin Oncol 2009;27:5868-73.

[3] Weeks JC, Catalano PJ, Cronin A, Finkelman MD, Mack JW, Keating NL, et al. Patients' expectations about effects of chemotherapy for advanced cancer. $N$ Engl J Med 2012;367:1616-25.

[4] Davis C. Drugs, cancer and end-of-life care: A case study of pharmaceuticalization? Social Science \& Medicine 2015;131:207-14. doi:10.1016/j.socscimed.2014.12.007.

[5] Miller K, Wang M, Gralow J, Dickler M, Cobleigh M, Perez EA, et al. Paclitaxel plus bevacizumab versus paclitaxel alone for metastatic breast cancer. $N$ Engl J Med 2007;357:2666-76.

[6] Schneider CK, Schäffner-Dallmann G. Typical pitfalls in applications for marketing authorization of biotechnological products in Europe. Nature Reviews Drug Discovery 2008;7:893-9. doi:10.1038/nrd2728.

[7] Bugat R, Guastalla JP, Misset JL. [Advanced ovarian cancer: a need for redefining therapeutic objectives and strategy]. Bull Cancer 2005;92:1003-6.

[8] Rothenberg ML, Carbone DP, Johnson DH. Improving the evaluation of new cancer treatments: challenges and opportunities. Nat Rev Cancer 2003;3:303-9.

[9] Sobrero AF, Pastorino A, Sargent DJ, Bruzzi P. Raising the Bar for Antineoplastic Agents: How to Choose Threshold Values for Superiority Trials in Advanced Solid Tumors. Clinical Cancer Research 2015;21:1036-43. doi:10.1158/1078-0432.CCR-14-1505.

[10] Pilz LR, Manegold C, Schmid-Bindert G. Statistical considerations and endpoints for clinical lung cancer studies: Can progression free survival (PFS) substitute overall survival (OS) as a valid endpoint in clinical trials for advanced non-small-cell lung cancer? Transl Lung Cancer Res 2012;1:26-35. doi:10.3978/j.issn.2218-6751.2011.12.08.

[11] Steer CB, Marx GM, Harper PG. Is there quality in clinical benefit? Ann Oncol 2001;12:1191-3.

[12] Watts S, Stenner P. Doing Q Methodological Research. Theory, Method \& Interpretation. SAGE Publications Ltd; 2012.

[13] Baker R, Thompson C, Mannion R. Q methodology in health economics. Journal of Health Services Research \& Policy 2006;11:38-45.

[14] Brown S. Q methodology and qualitative research. Qualitative Health Research 1996;4:561-7.

[15] Stephenson W. The study of behaviour: $Q$ technique and its methodology. Chicago: University of Chicago Press; 1953.

[16] Baker R, Wildman J, Mason H, Donaldson C. Q-ING FOR HEALTH-A NEW APPROACH TO ELICITING THE PUBLIC'S VIEWS ON HEALTH CARE RESOURCE ALLOCATION. Health Econ 2013. doi:10.1002/hec.2914. 
[17] Aldrich S, Eccleston C. Making sense of everyday pain. Social Science \& Medicine 2000;50:1631-41.

[18] Stenner PHD, Cooper D, Skevington SM. Putting the $Q$ into quality of life; the identification of subjective constructions of health-related quality of life using $Q$ methodology. Social Science \& Medicine 2003;57:2161-72. doi:10.1016/S02779536(03)00070-4.

[19] Morecroft C, Cantrill J, Tully MP. Individual patient's preferences for hypertension management: A Q-methodological approach. Patient Education and Counseling 2006;61:354-62.

[20] van Exel J, de Graaf G, Brouwer W. Care for a break? An investigation of informal caregivers' attitudes toward respite care using Q-methodology. Health Policy 2007;83:33242.

[21] Brouwer M. Q is accounting for tastes. Journal of Advertising Research 1999;39:35-9.

[22] Jeannin N, Blois Da Conceicao S, Protière C. [Reflections about the efficiency criteria for cancer treatments during marketing authorization application]. Bulletin Du Cancer 2013;100:259-69.

[23] Brown SR. Political subjectivity: Applications of Q methodology in political science. vol. Yale University Press. New Haven: 1980.

[24] Ramlo S. Centroid and Theoretical Rotation: Justification for Their Use in Q Methodology Research. Mid-Western Educational Researcher 2016;28.

[25] Abdi H. Factor rotations in factor analyses. Encyclopedia for Research Methods for the Social Sciences Sage: Thousand Oaks, CA 2003:792-795.

[26] Brown SR. A primer on Q methodology. Operant Subjectivity 1993;16:91-138.

[27] Baker RM. Economic rationality and health and lifestyle choices for people with diabetes. Social Science \& Medicine 2006;63:2341-2353.

[28] McHugh N, Baker RM, Mason H, Williamson L, van Exel J, Deogaonkar R, et al. Extending life for people with a terminal illness: a moral right and an expensive death? Exploring societal perspectives. BMC Medical Ethics 2015;16:14. doi:10.1186/s12910-0150008-x.

[29] Garattini S, Chalmers I. Patients and the public deserve big changes in evaluation of drugs. Bmj 2009;338:b1025.

[30] Mullard A. Mediator scandal rocks French medical community. The Lancet 2011;377:890-2.

[31] Baker R, van Exel J, Mason H, Stricklin M. Connecting Q \& surveys: three methods to explore factor membership in large samples. Operant Subjectivity 2010;34:38-58.

[32] French Sunshine Act - Is government backing down 20121025_FrenchSunshineAct.pdf n.d.

[33] Emmerich J, Dumarcet N, Lorence A. France's New Framework for Regulating OffLabel Drug Use. N Engl J Med 2012;367:1279-81.

[34] Silvestri G, Pritchard R, Welch HG. Preferences for chemotherapy in patients with advanced non-small cell lung cancer: descriptive study based on scripted interviews. British Medical Journal 1998;317:771-5.

[35] Mayor S. Time to raise the bar for cancer drug approval? The Lancet Oncology 2010;11:16-7. 


\begin{tabular}{|c|c|c|c|c|c|c|c|c|c|c|c|}
\hline & & \multicolumn{2}{|c|}{ F1 } & \multicolumn{2}{|c|}{$\mathbf{F 2}$} & \multicolumn{2}{|c|}{ F3 } & \multicolumn{2}{|c|}{ loaders } & \multicolumn{2}{|c|}{ Total } \\
\hline & & $\mathrm{n}$ & $\%$ & $\mathrm{n}$ & $\%$ & $\mathrm{n}$ & $\%$ & $\mathrm{n}$ & $\%$ & $\mathrm{n}$ & $\%$ \\
\hline & & 72 & & 18 & & 8 & & 98 & & 146 & \\
\hline \multirow[t]{5}{*}{ Population } & Health decision makers & 16 & 22 & 0 & 0 & 0 & 0 & 16 & 16 & 19 & 13 \\
\hline & Oncologists & 18 & 25 & 3 & 17 & 5 & 63 & 26 & 27 & 27 & 19 \\
\hline & Cancer patients & 13 & 18 & 11 & 61 & 2 & 25 & 26 & 27 & 52 & 36 \\
\hline & General population & 25 & 35 & 3 & 17 & 1 & 13 & 29 & 30 & 46 & 32 \\
\hline & $\begin{array}{l}\text { Members of the } \\
\text { pharmaceutical industry }\end{array}$ & 0 & 0 & 1 & 6 & 0 & 0 & 1 & 1 & 2 & 1 \\
\hline
\end{tabular}

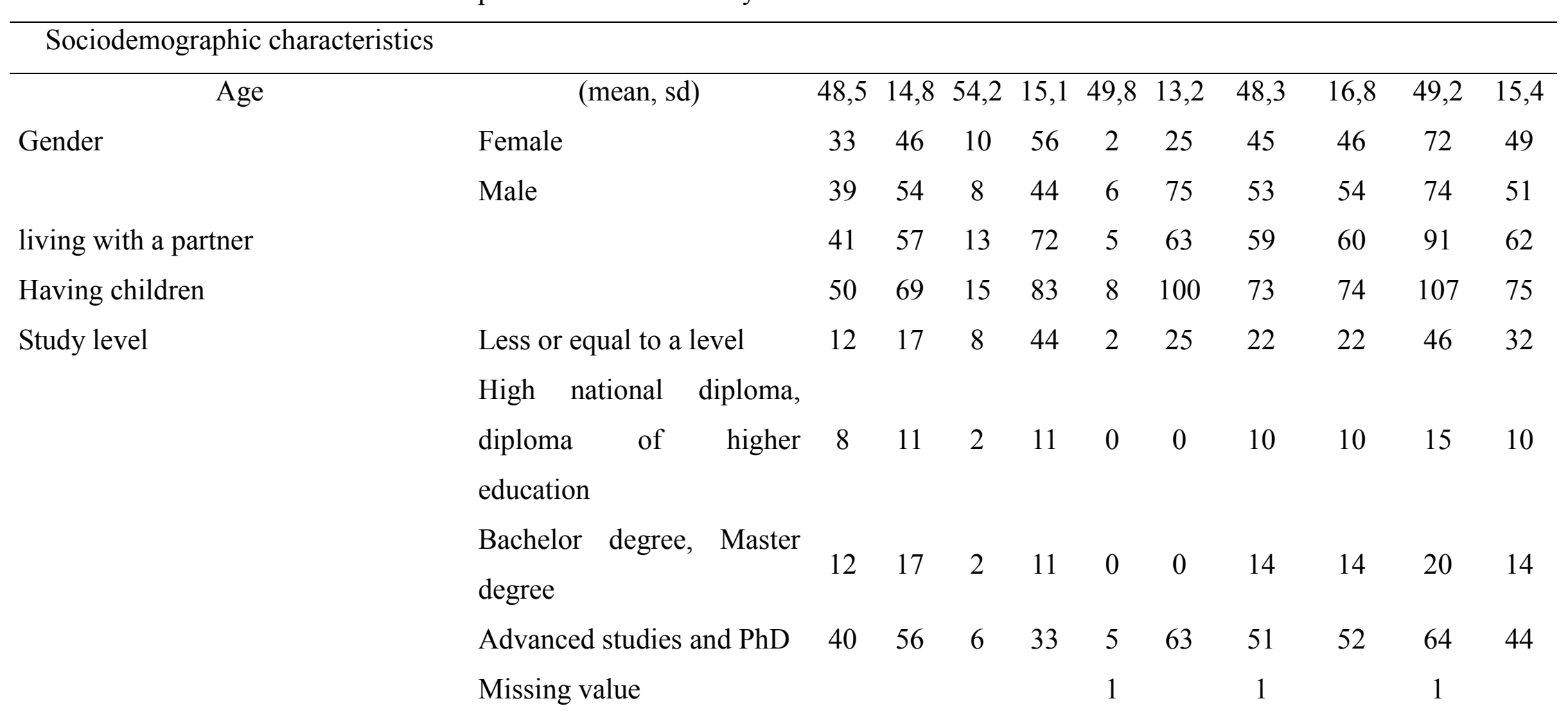




\begin{tabular}{|c|c|c|c|c|c|c|c|c|c|c|c|}
\hline \multirow[t]{6}{*}{ Professional status } & Employed & 44 & 61 & 10 & 56 & 7 & 88 & 61 & 62 & 81 & 55 \\
\hline & Unemployed & 6 & 8 & 0 & 0 & 0 & 0 & 6 & 6 & 9 & 6 \\
\hline & Students & 5 & 7 & 1 & 6 & 0 & 0 & 6 & 6 & 12 & 8 \\
\hline & Retired & 15 & 21 & 7 & 39 & 1 & 13 & 23 & 23 & 38 & 26 \\
\hline & Invalidity & 0 & 0 & 0 & 0 & 0 & 0 & 0 & 0 & 3 & 2 \\
\hline & Missing value & 2 & & & & & & 2 & & 3 & \\
\hline \multicolumn{12}{|l|}{ Cancer experience } \\
\hline \multicolumn{2}{|c|}{ Has already been treated for a cancer } & 19 & 26 & 11 & 61 & 2 & 25 & 32 & 33 & 59 & 41 \\
\hline \multicolumn{2}{|c|}{ Has already been treated for another serious illness } & 7 & 10 & 2 & 11 & 2 & 25 & 11 & 11 & 15 & 11 \\
\hline \multicolumn{2}{|c|}{ Has a close relative who has (had) a cancer } & 57 & 79 & 14 & 19 & 8 & 100 & 79 & 81 & 120 & 83 \\
\hline \multicolumn{2}{|c|}{$\begin{array}{l}\text { Has been confronted with the death of a close relative because of } \\
\text { cancer }\end{array}$} & 55 & 76 & 11 & 61 & 6 & 75 & 72 & 73 & 106 & 73 \\
\hline \multicolumn{2}{|c|}{ Length of the questionnaire } & 31,1 & 15,6 & 38,2 & 13,0 & 29,2 & 19,3 & 32,2 & 15,6 & 34,1 & 15,0 \\
\hline
\end{tabular}




\section{It's sufficient for a treatment to be at least as effective as the best available treatment, for receiving $M A^{I}$.}

2. An effective treatment is a treatment which allows the patient to live a comparable life as the one before the disease.

$2 \quad 1-3$

3. In my view a treatment that reduces the risk of disease progression must receive MA even if it temporarily affects thinking skills, $-1 \quad 1$ concentration and memory.

4. If a treatment represents real medical progress, it is legitimate that its cost for the society is very high.

5. For an illness where the average lifespan without treatment is 6 months, a survival gain of 1 month is a sufficient benefit for receiving a $-3 \quad-2$ MA.

6 It's important that patients are represented and that their opinions are taken into account in MA committee deliberations.

7. It's important that members of the general population are represented and that their views on treatment benefits are taken into account in $2 \quad-2 \quad-2$ MA committee deliberation

8. If standard treatment results in an average life expectancy of 1 year, a new treatment must produce a survival gain of at least 6 months to $0 \quad-3 \quad-4$ receive MA.

9. For illnesses where half of the patients will benefit from the treatment, a new treatment must produce a survival gain of at least 6 months $0 \quad 0-3$ to receive a MA. 
receive a MA.

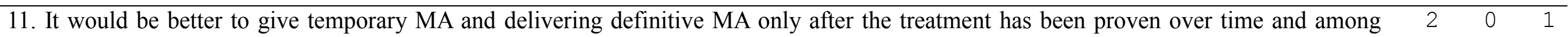
different populations.

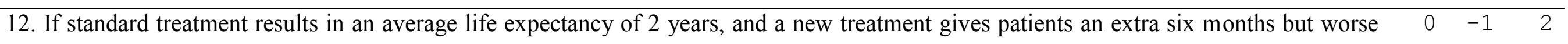
side effects, the new treatment must receive a MA.

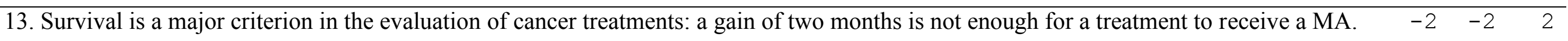

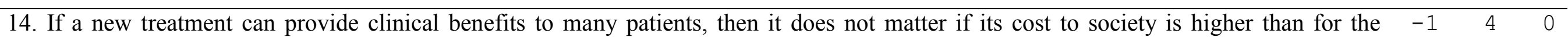
standard treatment.

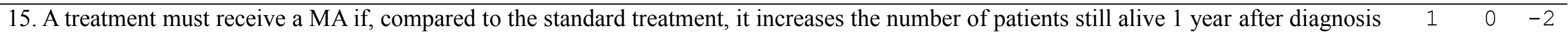
by at least $10 \%$.

16. A treatment with significant side effects must receive a MA if it prolongs lifespan of at least 1 year.

17. If a new treatment increases survival time from 24 to 34 months but the cost is multiplied by 50 it must be given MA.

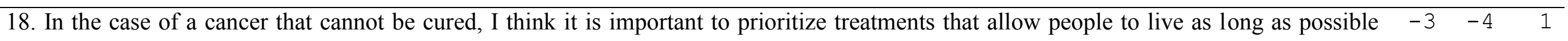
even if it implies an alteration of QoL.

19. If a treatment can prolong lifespan, even by one month, it should be given MA whatever its cost to society. 
and autonomy.

21. In case of cancer with little chance of cure, a treatment which reduces pain must receive MA even if it affects patients' abilities to reflect, 1 concentrate and remember.

22. A more expensive treatment which is not more effective in terms of life extension than the standard treatment, but which protects QoL 1 must receive a MA.

23. If a treatment which was intended to prolong my life prevented me from living a normal life, I would prefer not to take it.

$1 \quad 0 \quad-2$

24. In case of promising treatments for which there is a lack of evidence, it's important to give temporary MA in order to make them 1 accessible quickly.

25. A treatment that provides a slight benefit for an illness for which few treatments are available, must receive MA.

$0 \quad-1 \quad 4$

26. The treatment cost for society has to be considered during the MA procedure.

$2-4 \quad-1$

27. In my view, compared to the gain in survival, QoL is a secondary endpoint.

$\begin{array}{lll}-4 & -3 & -1\end{array}$

28. Treatments must be regularly re-evaluated after their launch on the market, to judge their efficacy and their tolerance in general practice.

$4 \quad 4 \quad 3$

29. I would be prepared to accept a treatment with significant side effects, even if the chances that it prolongs my life are low.

$\begin{array}{lll}-4 & -3 & 1\end{array}$

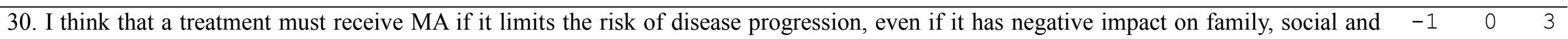
professional life.

31. It is important that outputs of the MA committee are transparent and made public. 
